\title{
BMJ Open Pain associated with psoriasis: systematic scoping review protocol
}

\author{
Ana Sanader Vucemilovic, ${ }^{1}$ Danijela Nujic, ${ }^{2}$ Livia Puljak (iD ${ }^{3}$
}

To cite: Sanader Vucemilovic A, Nujic D, Puljak L. Pain associated with psoriasis: systematic scoping review protocol. BMJ Open 2020;10:e031461. doi:10.1136/ bmjopen-2019-031461

- Prepublication history and additional material for this paper are available online. To view these files, please visit the journal online (http://dx.doi. org/10.1136/bmjopen-2019031461).

Received 06 May 2019 Revised 15 April 2020 Accepted 01 May 2020

\section{Check for updates}

\section{Author(s) (or their} employer(s)) 2020. Re-use permitted under CC BY-NC. No commercial re-use. See rights and permissions. Published by BMJ.

${ }^{1}$ Department of Dermatology, University Hospital Center Split Križine, Split, Croatia

${ }^{2}$ Department of Public Health, Josip Juraj Strossmayer University of Osijek School of Medicine, Osijek, OsijekBaranja, Croatia

${ }^{3}$ Center for Evidence-Based Medicine and Health Care, Catholic University of Croatia, Zagreb, Croatia

\section{Correspondence to}

Livia Puljak;

livia.puljak@unicath.hr; livia. puljak@gmail.com

\section{ABSTRACT}

Introduction Psoriasis is a common chronic skin inflammatory disease. Its presentation, apart from affected skin areas, involves other unpleasant symptoms, such as pain. Pain deteriorates the patient's quality of life, impairing their daily behaviour and functioning. Therefore, the alleviation of pain in patients with psoriasis should be one of the most desired outcomes of successful treatment. The aim of this study is to summarise available evidence about pain in patients with psoriasis using systematic scoping review methodology in order to map the relevant literature.

Methods and analyses Our scoping systematic review will provide evidence synthesis of the literature, both quantitative and qualitative, about the pain associated with psoriasis, including pain associated with psoriatic arthritis. Any types of studies will be eligible for inclusion, and we will not have any time, language or publication status restrictions. We will search MEDLINE, Embase and PsycINF0 via OVID, as well as Cochrane Central Register of Clinical Trials, Cochrane Database of Systematic Reviews via Cochrane Library, CINAHL via EBSC0, OpenGrey and ProQuest Dissertations and Theses Global. All databases will be searched from the date of their inception. Retrieved bibliographic records and potentially relevant full texts will be screened by two authors independently. Two researchers will extract data independently. Any discrepancies will be resolved via discussion or consultation of the third author, if necessary. To appraise studies, we will use a Mixed Methods Appraisal Tool, AMSTAR 2, Cochrane risk of bias tool and ROBINS. Our findings will be reported according to the Preferred Reporting Items for Systematic Reviews and MetaAnalyses Extension for Scoping Reviews.

Ethics and dissemination The proposed study will not be conducted with human participants. We will only use published data and therefore ethics approval is not required. Our findings will be disseminated via peerreviewed manuscript and conference reports.

\section{INTRODUCTION}

Psoriasis is a chronic inflammatory disease, also recognised as one of the most common immune-mediated diseases, ${ }^{1} \quad$ affecting $1 \%-3 \%$ of people worldwide. ${ }^{23}$ Common sites of involvement are scalp, extensor surfaces of the limbs, elbows and knees, sacral and genital regions, although it can also affect all other body areas. ${ }^{23}$ Aside from visible skin lesions, other related signs and symptoms can
Strengths and limitations of this study

- This will be a systematic scoping review examining pain associated with psoriasis.

- We will search multiple sources of information, including grey literature.

- Articles published at any time will be eligible; date of publication will be transparently reported and will allow a temporal analysis of the development of this research topic.

- Outcomes and outcome measures reported in the different studies can be incomparable; still, we will report them transparently to inform readers about the potential methodological differences and deficiencies of the studies involved.

- We will strive to include studies published in any language, but there is an option that we will not be able to enable the translation of all studies; this will be reported transparently.

have a strong impact on psychophysiological and social health. ${ }^{4}$ Studies have demonstrated that over $90 \%$ of patients with psoriasis report physical skin symptoms, ${ }^{5-7}$ among which are frequently skin pain and skin discomfort, ${ }^{89}$ with more than $40 \%$ of these patients expressing the presence of skin pain. ${ }^{10}$ Its impact on the quality of life, including body pain scores, can be comparable to that of other major medical conditions such as heart disease and diabetes. ${ }^{4}$

Pain is defined as an unpleasant sensory and emotional experience associated with actual or potential tissue damage or described in terms of such damage. ${ }^{11}$ One of the pain classifications found in literature categorises pain as nociceptive or neuropathic. ${ }^{12} 13$ Nociceptive pain is a result of actual or threatened damage of non-neural tissue and due to nociceptors activation, with normal functioning somatosensory nervous system. ${ }^{12}{ }^{13}$ Neuropathic pain, in contrast with nociceptive pain, is caused by a disease or lesion of the somatosensory nervous system. ${ }^{12} 14$

Besides skin pain, patients with chronic skin disorders, such as psoriasis, may also experience pain in other parts of the body. Pain that occurs in patients with psoriasis outside 
of the skin mostly affects joints and musculoskeletal areas and it probably occurs due to the association of psoriasis with different systemic comorbidities, including psoriatic arthritis. ${ }^{15}$ Although symptoms such as itching and scaling tend to be more common than pain in psoriasis, the recent study emphasised that skin pain was strongly associated with comorbid psychiatric and medical conditions. ${ }^{16}$ Skin pain in patients with psoriasis tends to have a great negative effect on patient's everyday life, including basic life needs, interpersonal relations ${ }^{16}{ }^{17}$ and work productivity. ${ }^{18}$

Considering reports indicating high prevalence and burden of pain in psoriasis, it is important to devote more attention to its epidemiology, diagnosis and management.

In the scientific literature, there is no available highquality evidence synthesis about the pain associated with psoriasis. Pithadia et al have published a review devoted solely to cutaneous pain associated with psoriasis; additionally, that was a narrative review and not a systematic review or systematic scoping review. ${ }^{19}$

The purpose of this systematic scoping review is to systematically summarise evidence from research literature about any type of pain in patients with psoriasis (skin pain, pain in psoriatic arthritis, and/or other types of acute and chronic pain).

\section{METHODS AND ANALYSES Study design}

This will be a systematic scoping review. We designed and will report this Scoping Reviews (ScR) in line with the Preferred Reporting Items for Systematic Reviews and Meta-Analyses (PRISMA). ${ }^{20}$

\section{Protocol and registration}

We developed this protocol a priori, and by submitting it to an open-access peer-reviewed journal, we would like to ensure its public accessibility and transparency of our methods.

\section{Patient and public involvement}

We did not involve patients or the public in our work.

\section{Eligibility criteria}

We will include any type of studies on humans that have analysed any data related to pain associated with psoriasis. We will not use any restrictions regarding period, language or type of publication. We will exclude studies about pruritus because pain and pruritus are not the same symptoms.

\section{Information sources}

We will search the following information sources: MEDLINE, Embase and PsycINFO via OVID, as well as Cochrane Central Register of Clinical Trials (CENTRAL), Cochrane Database of Systematic Reviews (CDSR) via Cochrane Library, CINAHL via EBSCO, OpenGrey and ProQuest Dissertations and Theses Global. All databases will be searched from the date of their inception. After we identify eligible studies based on the search of those databases, we will additionally analyse all references and citations of those studies, to potentially find additional relevant studies. The date of the most recent search will be noted. All search results will be exported to EndNote citation software (EndNote, Clarivate Analytics, Philadelphia, PA, USA); duplicates will be removed via software, and then manually if needed.

\section{Search}

A full search strategy for the selected databases is shown in online supplementary appendix 1 . Our search strategies were created using a combination of various keywords for psoriasis and pain, using both controlled vocabulary and free text searching.

\section{Selection of sources of evidence}

Screening, that is, selection of studies, will be conducted in two stages. First, bibliographic records obtained via database searching will be screened by two authors independently (ASV and DN). All records retained as relevant or potentially relevant will be obtained in full text. Second, all retrieved full texts will be screened by two authors independently (ASV and DN). Records retrieved via searching of citations and references of eligible studies will also be screened by the same two authors independently. Discrepancies in opinion regarding study eligibility will be resolved by discussion, and-if necessary, in consultation with the third author (LP). We will report which studies were excluded on the level of fulltext assessments, with reasons.

\section{Data charting process}

Two authors (ASV and DN) will develop the data extraction form. This form will determine which variables to extract, and how. The form will be calibrated on 20 randomly selected studies. During the calibration exercise, the authors will independently extract the data, and then discuss results; we will update the form in an iterative process, if necessary. The final version of the data extraction form will be enclosed with the full report at the time of completion of this review.

\section{Data items}

We will extract the following items from eligible studies: name of the first author, country of authors' affiliations, year of publication, journal, aim, country where the study was conducted, study design, size of the study sample, type of study sample (ie, any type of psoriasis, or specific types of psoriasis), setting, outcomes and outcome measures analysed, numerical results reported, type of pain (cutaneous, psoriatic arthritis pain and pain in general) data on pain prevalence, pain intensity, quality, location and duration, and conclusions regarding pain in the context of psoriasis. We will also extract data on conflict of interest of authors of the eligible studies, as well as the type of funding.

Two researchers (ASV and DN) will extract data independently. Any discrepancies in data extraction will be 
resolved by discussion or consultation with the third author (LP) if necessary.

\section{Critical appraisal of individual sources of evidence}

If we find any systematic reviews on this topic, we will assess them with the second version of A MeaSurement Tool to Assess systematic Reviews (AMSTAR-2), a tool for appraising systematic reviews. ${ }^{21}$ If we find any randomised controlled trials or non-randomised studies of interventions, the risk of bias will be appraised using Cochrane risk of bias (RoB) tool ${ }^{22}$ or risk of bias tool to assess nonrandomized studies of interventions (ROBINS), ${ }^{23}$ respectively. To appraise quantitative and qualitative studies, we will use a Mixed Methods Appraisal Tool, version 2018. ${ }^{24}$

\section{Synthesis/presentation of results}

We do not expect to find data relevant for conducting a meta-analysis; meta-analysis is not anticipated a priori in scoping reviews. ${ }^{20}$ All information we find about the pain associated with psoriasis will be categorised and narrative evidence synthesis will be conducted. If possible, we will try to quantitatively summarise the extracted information.

\section{Selection of sources of evidence}

In our results, we will report numbers of sources of evidence, screened, assessed for eligibility, and included in the systematic scoping review, with reasons for exclusions at each stage, via a flow diagram, that is, PRISMA flow chart. $^{20}$ We will provide elaborate information for included studies in a table titled 'Characteristics of included studies', as well as a list of excluded studieswith reasons and references.

\section{Characteristics of sources of evidence}

For each included study, we will present the following characteristics: studies' place of origin, aims, design, methods and potentially other relevant information.

\section{Ethics and dissemination}

The proposed study will not recruit or collect data directly from human participants. We will only use published data and therefore ethics approval is not required. Our findings will be disseminated via peer-reviewed manuscript and conference reports.

\section{DISCUSSION}

With this systematic review, we expect to create a comprehensive evidence synthesis about pain reported as associated with psoriasis. We are aware that we will surely find heterogeneous types of studies and pain-related topics within the context of psoriasis, but such heterogeneity is inherent in scoping reviews; this is highlighted in the introductory parts of the PRISMA-ScR ('summarise findings from a body of knowledge that is heterogeneous in methods or discipline'). ${ }^{20}$ Our eligibility criteria will allow for the inclusion of older articles. However, this will be transparently reported and will allow us to clearly depict the evolution of this research topic. Conducting the proposed scoping review will enable us to provide evidence mapping and conduct of future focused systematic reviews.

The strength of this study is that this will be the first systematic scoping review on the proposed topic. Additionally, we chose to search multiple information sources, which should enable effective literature searching. There are no uniform guidelines for sources that should be searched in scoping reviews because this ultimately depends on the topic. For example, PRISMA extension for scoping reviews simply asks authors to transparently report which information sources were searched and encourages authors to report search strategy for at least one database. ${ }^{20}$ Instructions in this and other guidance provide only the following suggestion 'The search strategy should be comprehensive in order to identify both published and unpublished (grey literature) literature'. Joanna Briggs Institute manual for scoping reviews recommends searching 'at least two appropriate online databases relevant to the topic'. ${ }^{25}$ Due to the lack of uniform guidance, we have suggested using a very comprehensive search strategy, which involves six databases and two sources of grey (unpublished) literature.

\section{Expected timeline}

The authors will not start working on the conduct of this scoping systematic review until the publication of this protocol.

\section{Twitter Livia Puljak @liviapuljak}

Contributors Study idea conception: LP. Study design: LP, DN and ASV. Writing of the protocol: LP, DN and ASV. Approval of the final version: LP, DN and ASV. Study conduct, acquisition of data, analysis and interpretation of data: not applicable; this manuscript describes a study protocol.

Funding The authors have not declared a specific grant for this research from any funding agency in the public, commercial or not-for-profit sectors.

Competing interests None declared.

Patient and public involvement Patients and/or the public were not involved in the design, or conduct, or reporting or dissemination plans of this research.

Patient consent for publication Not required.

Provenance and peer review Not commissioned; externally peer reviewed.

Open access This is an open access article distributed in accordance with the Creative Commons Attribution Non Commercial (CC BY-NC 4.0) license, which permits others to distribute, remix, adapt, build upon this work non-commercially, and license their derivative works on different terms, provided the original work is properly cited, appropriate credit is given, any changes made indicated, and the use is non-commercial. See: http://creativecommons.org/licenses/by-nc/4.0/.

ORCID iD

Livia Puljak http://orcid.org/0000-0002-8467-6061

\section{REFERENCES}

1 Griffiths CE, Barker JN. Pathogenesis and clinical features of psoriasis. Lancet 2007;370:263-71.

2 Greaves MW, Weinstein GD. Treatment of psoriasis. N Engl J Med 1995;332:581-9.

3 Menter A, Gottlieb A, Feldman SR, et al. Guidelines of care for the management of psoriasis and psoriatic arthritis: section 1. overview of psoriasis and guidelines of care for the treatment of psoriasis with biologics. J Am Acad Dermatol 2008;58:826-50. 
4 Rapp SR, Feldman SR, Exum ML, et al. Psoriasis causes as much disability as other major medical diseases. J Am Acad Dermatol 1999;41:401-7.

5 Finlay AY, Khan GK, Luscombe DK, et al. Validation of sickness impact profile and psoriasis disability index in psoriasis. $\mathrm{Br} \mathrm{J}$ Dermatol 1990;123:751-6.

6 Gowda S, Goldblum OM, McCall WV, et al. Factors affecting sleep quality in patients with psoriasis. J Am Acad Dermatol 2010;63:114-23.

7 Fortune DG, Richards HL, Griffiths CEM. Psychologic factors in psoriasis: consequences, mechanisms, and interventions. Dermatol Clin 2005;23:681-94.

8 Sampogna F, Gisondi P, Melchi CF, et al. Prevalence of symptoms experienced by patients with different clinical types of psoriasis. $\mathrm{Br} \mathrm{J}$ Dermatol 2004;151:594-9.

9 McKenna KE, Stern RS. The impact of psoriasis on the quality of life of patients from the 16-center PUVA follow-up cohort. J Am Acad Dermatol 1997;36:388-94.

10 Marinus J, Moseley GL, Birklein F, et al. Clinical features and pathophysiology of complex regional pain syndrome. Lancet Neurol 2011:10:637-48.

11 Unaeze J, Nijsten T, Murphy A, et al. Impact of psoriasis on healthrelated quality of life decreases over time: an 11-year prospective study. J Invest Dermatol 2006;126:1480-9.

12 lasp-pain.org. IASP Terminology - IASP, 2020. Available: <https:// www.iasp-pain.org/Education/Content.aspx?ItemNumber=1698> [Accessed 25 Mar 2020].

13 Nicholson B. Differential diagnosis: nociceptive and neuropathic pain. Am J Manag Care 2006;12:S256-62.

14 Jensen TS, Finnerup NB. Allodynia and hyperalgesia in neuropathic pain: clinical manifestations and mechanisms. Lancet Neurol 2014;13:924-35

15 Thom N, Ritchlin CT, Zhang X, et al. Prevalence of chronic axial pain, inflammatory back pain, and spondyloarthritis in diagnosed psoriasis. Arthritis Care Res 2015;67:829-35.
16 Korman NJ, Zhao Y, Pike J, et al. Increased severity of Itching, pain, and scaling in psoriasis patients is associated with increased disease severity, reduced quality of life, and reduced work productivity. Dermatol Online J 2015;21. [Epub ahead of print: 16 Oct 2015].

17 Riol-Blanco L, Ordovas-Montanes J, Perro M, et al. Nociceptive sensory neurons drive interleukin-23-mediated psoriasiform skin inflammation. Nature 2014;510:157-61.

18 Ljosaa TM, Mork C, Stubhaug A, et al. Skin pain and skin discomfort is associated with quality of life in patients with psoriasis. $J$ Eur Acad Dermatol Venereol 2012;26:29-35.

19 Pithadia DJ, Reynolds KA, Lee EB, et al. Psoriasis-Associated cutaneous pain: etiology, assessment, impact, and management. $J$ Dermatolog Treat 2019;30:435-40.

20 Tricco AC, Lillie E, Zarin W, et al. PRISMA extension for scoping reviews (PRISMA-ScR): checklist and explanation. Ann Intern Med 2018;169:467-73.

21 Shea BJ, Reeves BC, Wells G, et al. AMSTAR 2: a critical appraisal tool for systematic reviews that include randomised or nonrandomised studies of healthcare interventions, or both. $B M J$ 2017;358:j4008.

22 Higgins JPT, Altman DG, Gøtzsche PC, et al. The Cochrane collaboration's tool for assessing risk of bias in randomised trials. BMJ 2011;343:d5928.

23 Sterne JA, Hernán MA, Reeves BC, et al. ROBINS-I: a tool for assessing risk of bias in non-randomised studies of interventions. BMJ 2016;355:i4919.

24 Pluye PRE, Cargo M, Bartlett G, et al. Proposal: a mixed methods appraisal tool for systematic mixed studies reviews. J Montréal: McGill University, 2011: 2. 1-8. http://mixedmethodsappraisaltoo Ipublic.pbworks.com/w/file/fetch/84371689/MMAT\%202011\% 20criteria\%20and\%20tutorial\%202011-06-29updated2014.08.21. pdf

25 Aromataris EMZ. Joanna Briggs Institute reviewer's manual. The Joanna Briggs Institute 2017;2017. 